Review

\title{
Review of Computational Methods on Brain Symmetric and Asymmetric Analysis from Neuroimaging Techniques
}

\author{
P. Kalavathi ${ }^{1}$, M. Senthamilselvi ${ }^{1}$ and V. B. Surya Prasath ${ }^{2, *}$ \\ 1 Department of Computer Science and Applications, Gandhigram Rural Institute, Deemed University, \\ Gandhigram, 624302 Tamil Nadu, India; pkalavathi.gri@gmail.com (P.K.); kmt.selvi@gmail.com (M.S.) \\ 2 Computational Imaging and VisAnalysis (CIVA) Lab, Department of Computer Science, \\ University of Missouri-Columbia, Columbia, MO 65211, USA \\ * Correspondence: prasaths@missouri.edu; Tel.: +1-573-882-8391
}

Academic Editors: Yudong Zhang and Zhengchao Dong

Received: 26 November 2016; Accepted: 17 April 2017; Published: 18 April 2017

\begin{abstract}
The brain is the most complex organ in the human body and it is divided into two hemispheres-left and right. The left hemisphere is responsible for control of the right side of our body, whereas the right hemisphere is responsible for control of the left side of our body. Brain image segmentation from different neuroimaging modalities is one of the important parts of clinical diagnostic tools. Neuroimaging based digital imagery generally contain noise, inhomogeneity, aliasing artifacts, and orientational deviations. Therefore, accurate segmentation of brain images is a very difficult task. However, the development of accurate segmentation of brain images is very important and crucial for a correct diagnosis of any brain related diseases. One of the fundamental segmentation tasks is to identify and segment inter-hemispheric fissure/mid-sagittal planes, which separate the two hemispheres of the brain. Moreover, the symmetric/asymmetric analyses of left and right hemispheres of brain structures are important for radiologists to analyze diseases such as Alzheimer's, autism, schizophrenia, lesions and epilepsy. Therefore, in this paper, we have analyzed the existing computational techniques used to find brain symmetric/asymmetric analysis in different neuroimaging techniques such as the magnetic resonance (MR), computed tomography (CT), positron emission tomography (PET), single-photon emission computed tomography (SPECT), which are utilized for detecting various brain related disorders.
\end{abstract}

Keywords: computational imaging; midsagittal plane; inter-hemispheric fissure; symmetry analysis; neuroimaging

\section{Introduction}

The human brain exists inside a tough bone structure called the skull. It gives us awareness of ourselves and of our environment, processing a constant stream of sensory data and also controls our muscle movements, the secretions of our glands, and even our breathing and internal temperature [1]. The brain is made of three main parts: the forebrain, midbrain and hindbrain. The forebrain consists of the cerebrum, thalamus and hypothalamus. The midbrain has the tectum and tegmentum. The hindbrain is made of the cerebellum, pons and medulla. The midbrain, pons, and medulla are referred to together as the brainstem. The cerebrum or cortex is the largest part of the human brain, associated with higher brain function such as thought and action. The cerebral cortex is divided into four sections called lobes: the frontal lobe, parietal lobe, occipital lobe, and temporal lobe [2]. Different anatomical parts of the human brain are shown in Figure 1. Figure 1a shows a normal adult human brain along with a inter-hemispheric fissure (also known as cerebral or 
longitudinal fissure), and Figure $1 \mathrm{~b}$ shows important brain anatomical regions from a sagittal cross section of a magnetic resonance (MR) image.

There are many imaging modalities that are used in medical imaging technologies and, in particular, for imaging the brain. The most commonly used modalities are X-ray, Computed Tomography (CT), Positron Emission Tomography (PET), Single-Photon Emission Computed Tomography (SPECT), Ultrasound (US) and Magnetic Resonance (MR) imaging. Among them, CT and MR imaging are the most widely used imaging techniques for human brain clinical diagnosis and analysis. Recent advances in automatic image processing techniques enabled us to analyze the differences and similarities between the two brain hemispheres, compartment and intracranial structure to detect various brain abnormalities and differences in anatomical structures [3,4].

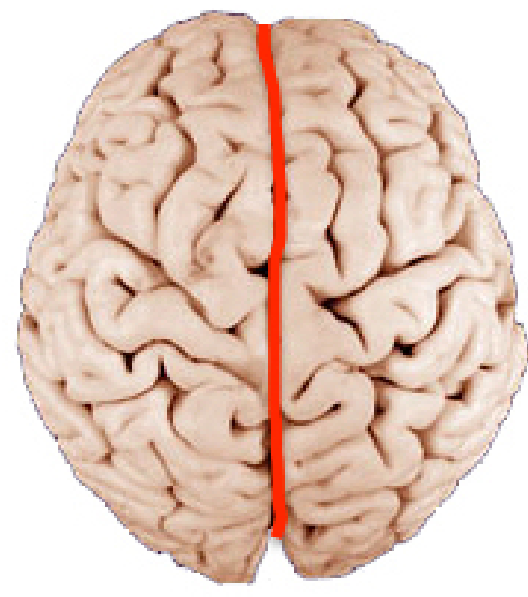

(a)

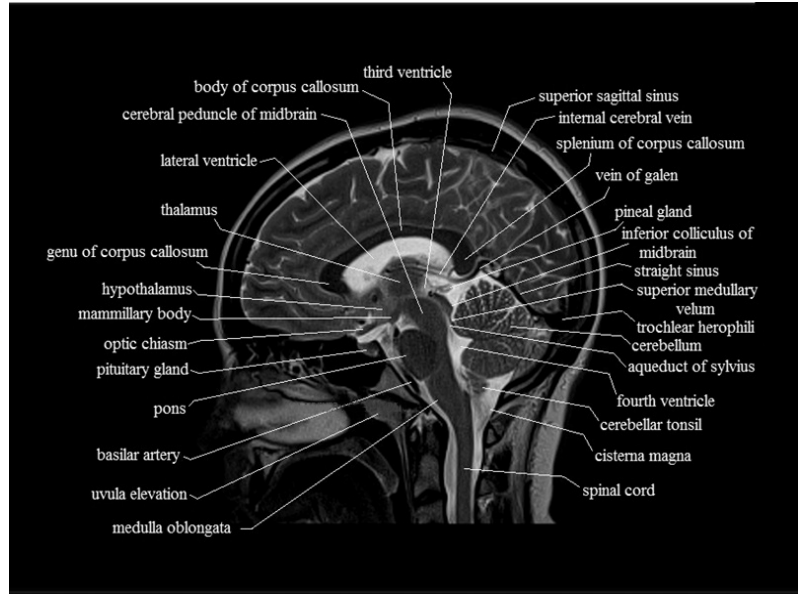

(b)

Figure 1. (a) inter-hemispheric fissure in a normal human brain; and (b) different brain anatomical structures from sagittal cross-section in a magnetic resonance (MR) image (courtesy of [5]).

The human brain exhibits an approximately bilateral symmetry across the sagittal plane. A longitudinal fissure separates the human brain into two distinct cerebral hemispheres. Identification of inter-hemispheric fissure (IHF) or midsagittal plane (MSP) of a brain image is required in clinical applications. MSP detection is one of the methods to segment left and right hemispheres. The MSP based brain hemisphere segmentation is based on the assumption that the human brain has a roughly symmetric structure with respect to the MSP. Therefore, most of the existing methods use several kinds of approaches and techniques to detect MSP. However, detecting the MSP is very difficult due to the poor visibility of the inter-hemispheric fissure. To overcome this limitation, skull symmetry as a constraint has been included as an important anatomical feature in MSP detection [6]. Segmented hemispheres has been applied to assess brain symmetries/asymmetries [7] to elucidate functional brain lateralization [8], natural brain development and neuro-degeneration [9], in addition to the effects of diseases on the human brain such as Alzheimer's disease [10], autism [11], schizophrenia [12,13], visual pathways disease [14], hearing loss [15], and lesions. More recently, computer-assisted methods have been developed for identification of multiple sclerosis and lesions from magnetic resonance (MR) brain scans, and also for the extraction of the cerebral ventricles in Schizophrenia studies.

Due to the aforementioned limitations in MSP based brain hemisphere segmentation, other computational methods and approaches have also been developed in recent times, e.g., fractal dimension [16], graph-cut algorithm [17], shape bottleneck algorithm [18], etc., in order to segment the left and right hemispheres for brain symmetric/asymmetric analysis. In the following section, we describe and review in detail the existing computational techniques developed and used for brain symmetric/asymmetric analysis to detect, analyze and treat various human brain disorders. These existing methods are categorized into two major classifications, and they are represented in Figure 2. 


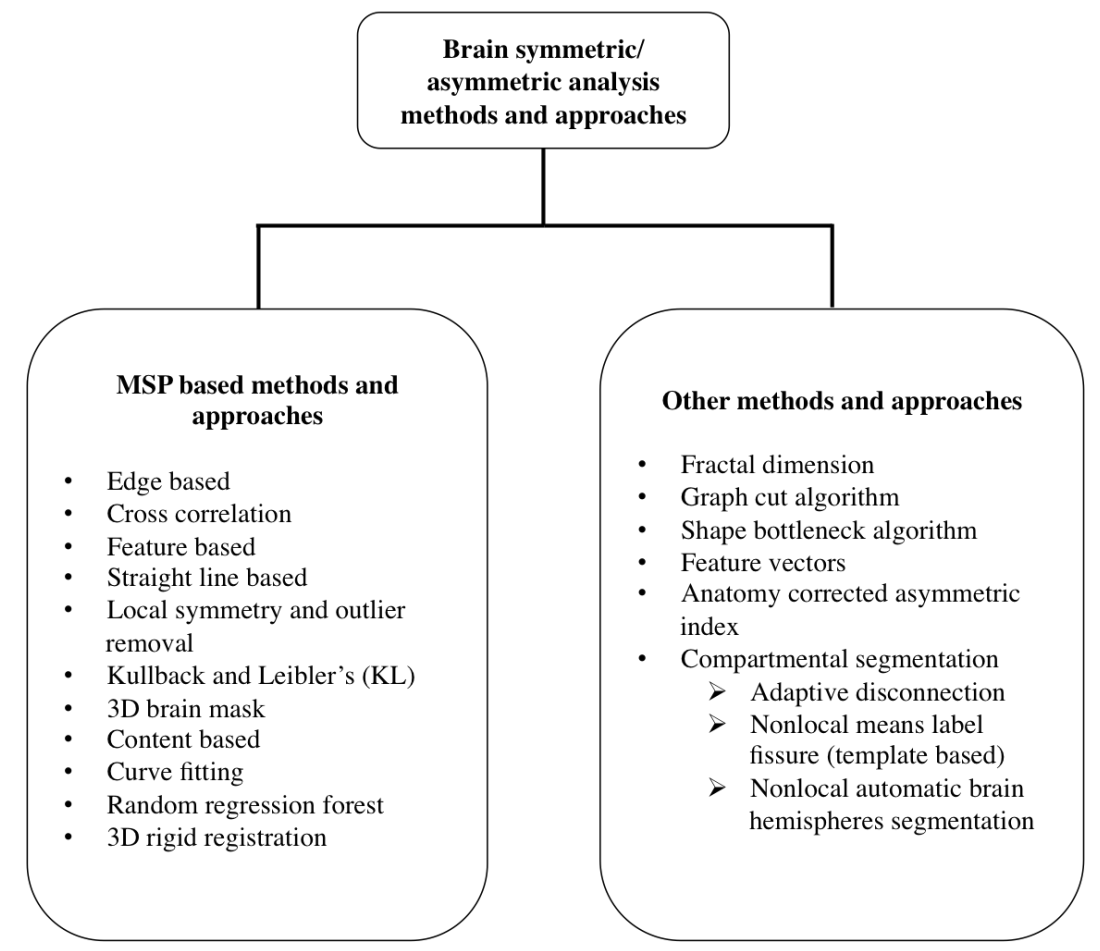

Figure 2. Classification of brain symmetric/asymmetric analysis methods and approaches.

\section{Review of Brain Symmetric/Asymmetric Analysis Methods}

\subsection{MSP Based Methods and Approaches}

The existing methods for MSP detection can further be classified into feature based, edge based, cross correlation based, search based, straight line based, local symmetry and outlier based, Kullback and Leibler (KL) based, 3D mask method based and content based approaches.

In the feature-based approach, the aim is to directly determine the inter-hemispheric fissure from its intensity and textural features. Brummer [19] proposed a 3D extension of Hough transform by observing that MSP appears as long lines in the coronal view, and this approach involves detection of lines from the edge maps of 2D brain images and then detecting MSP by fitting a plane in MRI brain volumes. Sometimes, the longitudinal fissure is no longer adequately described by a single plane because of the excessive amounts of central fluid sulcus, and more efficient algorithms are needed to detect the MSP.

In the symmetry-based approach [20], the MSP is defined as the plane that maximizes the similarity between the brain and its reflection. Symmetry based approaches first define a parameter space and then need to describe the MSP based on similarity measurement, such as the cross-correlation method which is used to assess the inter-hemispheric symmetry in the selected feature space and the intensity/edge based in addition to a search method/search criteria to find the parameters that maximize the similarity measures. The main drawback of the symmetry based approach is sensitivity to brain asymmetries and increased computational costs. The main advantage of this method is generalizability and adaptability to other medical image modalities, such as CT and PET.

Ardekani et al. [21] proposed an automatic method to detect the MSP in 3D MR brain and PET brain images. This line fitting/MSP straight-line algorithm seeks the plane with respect to which the image that exhibits the maximum symmetry and is measured by the cross-correlation between the images sections lying on either side. The search for the plane with maximum symmetry is performed by using a multiresolution approach that substantially decreases computational time. The choice of the starting plane was found to be an important issue in optimization and the method is tested on brain 
images from various imaging modalities in both human and animals. However, it does not produce satisfactory results when applied to a large number of clinical images.

A method for extraction of the ideal Mid-Sagittal Plane (iMSP) [22] for normal and pathological asymmetry brain images uses an edge-based and cross-correlation approach to decompose the plane fitting problem into the discovery of 2D symmetry axes on each slice, followed by a robust estimation of 3D plane parameters. The iMSP extraction algorithm was evaluated for capturing the iMSP from 3D normal and pathological neural images, and the algorithm is quantitatively measured by the input image offsets and image noise. The main challenges are the drastic structural asymmetry that often exists in pathological brains, and no isotropic data sampling that is common in clinical practice. It is found that the algorithm can extract the iMSP from input 3D images with the large asymmetrical lesions, arbitrary initial yaw, roll angle errors and low signal-to-noise level. The algorithm was also tested in PET and SPECT brain images.

The human brain is never perfectly symmetric and the MSP is not always a straight line, and, even for normal brains, their inter-hemispheric surfaces are curved. Therefore, with the assumption of MSP as a curved line, Prima et al. [23] developed an iterative approach to find MSP. This method worked by assuming an initial guess of the MSP and updating it by computing the local similarity measures between the two sides of the head by applying the block matching procedure in all types of brain imaging modalities like MRI, CT, PET and SPECT. However, this method does not work in functional MR and ultrasound brain images.

Linear stereotaxic registration [24] can also be used to extract the MSP in MR images of different subjects and are linearly transformed to match a common template image, whose MSP is the longitudinal median plane of the stereotaxic space. The MSP in this method is defined as a plane formed from the inter-hemispheric fissure line segments having the dominant orientation. The MSP detection method developed in [25] obtained a best plane based on the degree of similarity between the image and its reflection with respect to the plane. In each iteration, the best plane is identified by maximizing the similarity measure from brain MR images (see also [26]).

A rapid algorithm for automatic extraction of the MSP of the human cerebrum from normal and pathological neuroimages based on local symmetry and histogram outlier removal techniques was developed by Hu and Nowinski [27]. In this method, the MSP is detected by a line fitting algorithm in brain MR and CT images. However, more extensive analysis has not been done yet in all CT and MR morphological cases brain images.

Ekin [28] used a random sample consensus (RANSAC) method to detect MSP from its intensity and textural features in MRI brain images. It has found that 3D MR data is first processed as 1D image lines, then as 2D slices, and finally 3D volume. This makes it possible to detect an MSP quickly and robustly. However, to detect an MSP, the algorithm requires the availability of axial Proton Density (PD) contrast images. Because PD is one of the common contrasts in a typical brain MR scan, this is not a very limiting condition. MSP extraction based on the calculation of the Kullback and Leibler (KL) measures proposed by Volkau et al. [29] characterize the difference between two distributions. The slices along the sagittal direction are analyzed with respect to a reference slice and determined the coarse MSP. To calculate the final MSP, a local search algorithm is applied. They assume that the entropy of MSP is lower than that of the neighboring sagittal slices due to its large amount of cerebrospinal fluid (CSF). In their method, a volume of interest (VOI) is defined around the central slice in the sagittal direction and the KL measure is computed on all sagittal slices comparing each to the first slice of the VOI. By taking the slice that gives the maximum KL measure as the central plane for a new smaller VOI, a new search is performed until the MSP is estimated from MRI and CT brain images.

A method to segment T1-weighted MRI brain volumes into left and right cerebral hemispheres using the Graph Cuts algorithm is developed by Liang et al. [30]. The Graph Cuts algorithm compares the results of graph cuts segmentations against gold standard manual segmentations and with three popular software packages Brain Visa, CLASP, and Surf Relax. Song et al. [31] 
determined the MSP based on a group of assistant parallel lines and correlation of gravitational forces to detect the pathological brain in MRI. It also performs symmetric analysis in 2D slices followed by quantification for the two hemispheres. The hemispheres are partitioned by the geometry symmetry axis (GSA) based on the correlation to the gray level distribution (GLS). The quantification results are considered as a feature to distinguish the normal and abnormal brain slices. Liu et al. [32] successfully identified the MSP by minimizing the statistical dissimilarity between paired regions in opposing hemispheres and formulating the MSP extraction as an optimization problem. This method computes matrices for the left and right hemispheres that were treated as two feature vectors and found that the pathological brains are significantly more asymmetric and the variation of asymmetry degree is much wider. It also reported that there are significant gender-related asymmetry differences in MR brain images.

Bergo et al. [33] developed a heuristic maximization method to detect the MSP, which is fast and robust with 3D MRI brain imaging. It is assumed that the MSP contains the maximum area of Cerebrospinal Fluid (CSF) when ventricles are excluded. This method creates a 3D brain mask that excludes ventricles. The CSF score of each sagittal plane is obtained by computing the mean voxel intensity in the intersection between the plane and the brain mask. The plane with reasonably large brain mask intersection and minimal intensity score is taken as the best candidate for the MSP. Then, the CSF score is again calculated for all small transformations of the chosen plane and the plane with the lowest score is considered to be the final MSP. Zhao et al. [34] developed an unsupervised method to detect the inter-hemispheric metabolic asymmetry by calculating an anatomy corrected asymmetry index (ACAI) of the investigated image and effectively avoiding the impact from the asymmetric structure of the brain. The basic idea of the ACAI method is to take advantage of the anatomical information obtained from MRI, and construct an asymmetry indices (AI) map based on the classification of voxels.

Ruppert et al. [20] proposed a new symmetry based method for MSP in neuroimages which relies on image features detected using 3D Sobel edge operator and multi-scale correlation to extract the optimal MSP. This method is sensitive to image noises and deformations. A method proposed by Teverovskiy and $\mathrm{Li}$ [35] is different from the traditional intensity based cross correlation technique in that it performs the cross correlation on an edge image in order to capture the anatomical structures of the brain and skull while ignoring intensity fluctuation and found the MSP accurately on certain pathological images. However, the results could be severely affected when the initial estimate of the MSP is computed on a lower brain slice. In order to avoid it, Jayasuriya and Liew [16] developed an intensity based reflection approach to find the MSP in 3D MRI brain images that can easily be extended to different imaging modalities. However, besides being computationally demanding, intensity based reflection approach is highly sensitive to the asymmetry caused by various brain pathologies. Qi et al. [36] developed an automated computer aided ideal midline estimation system using a two step process. First, a Slice Selection Algorithm (SSA) is applied to automatically select an appropriate subset of slices from a large number of raw CT images. Next, an ideal midline detection is implemented on the selected subset of slices based on edge detection and Hough transform.

Favretto et al. [37] developed an automatic method for 3D rigid registration of MR brain images. This method is combined with brain segmentation and a greedy search algorithm to find the best match between the source and target MR brain images. Then, the MSP was found by using a heuristic search approach in the brain images. An automation method to find the MSP based on the KL measure from MR brain images was devised in Kuijf et al. [38]. The MSP is identified by initializing a surface that was deformed to represent the midsagittal surface. Wu et al. [39] developed a more accurate, efficient and robust MSP detection method based on 3D scale-invariant feature transform (SIFT) features, which are detected, clustered and indexed under a novel parallel framework. The GPU-K Dimensional tree algorithm was then used to validate on both synthetic and in vivo datasets having normal and pathological cases. Unlike the existing MSP extraction methods, this method mainly relies on the gray 
similarity, 3D edge registration and parameterized surface matching to determine the fissure plane (see also [40]).

A method to automatically detect the Anterior Commissure (AC), Posterior Commissure (PC), and MSP in T1-Weighted MR brain scans using the random regression forests method was developed by Liu and Dawant [41]. This method was evaluated using a leave-one-out approach with 100 clinical T1-weighted MR images and was compared with state-of-art methods including an atlas based approach with six nonrigid registration algorithms and a model based approach for the AC and PC segmentation, and a global symmetry based approach for the MSP.

Automatic segmentation of cerebral hemispheres using curve fitting was developed by Kalavathi and Prasath [42]. In this method, the MSP was detected as a curve, and is used to segment the left and right hemispheres. This method was tested using T1, T2 and PD weighted MR brain images.

Brain symmetry/asymmetry analysis using MSP based methods and approaches along with the technique used and image modalities applied are summarized in Table 1.

Table 1. Summary of the existing mid-sagittal plane (MSP) based methods and approaches for brain symmetric and asymmetric analysis.

\begin{tabular}{ccc}
\hline Methods & Techniques Used & Image Modality \\
\hline$[19]$ & Hough transformation & MRI \\
{$[21]$} & Line fitting algorithm & MRI \\
{$[22]$} & Edge and cross correlation methods & MRI, PET, SPECT \\
{$[23]$} & Block matching procedure & MRI, CT, PET, SPECT \\
{$[24]$} & Linear stereotaxic registration and template matching & MRI \\
{$[25]$} & Based on similarity measures & MRI \\
{$[27]$} & Local symmetry histogram based outlier removal & MRI, CT \\
{$[28]$} & Feature based approach & 2D and 3D MRI \\
{$[29]$} & Kullback-Leibler measure & MRI, CT \\
{$[30]$} & Graph cuts algorithm & T1-weighted MRI \\
{$[31]$} & Parallel line fitting and correlation & MRI \\
{$[32]$} & Similarity measure and optimization technique & MRI \\
{$[33]$} & Heuristic maximization method & 3D MRI \\
{$[34]$} & Anatomy corrected asymmetry index (ACAI) & FDG-PET \\
{$[20]$} & Edge based technique and multi scale correlation & 3D MRI, CT \\
{$[35]$} & Intensity based cross correlation approach & 3D MRI \\
{$[16]$} & Intensity based reflection approach & MRI, CT, PET, SPECT \\
{$[36]$} & Edge based and Hough Transformation method & CT \\
{$[37]$} & 3D rigid registration method, greedy search algorithm & MRI \\
{$[38]$} & Kullback-Leibler measure, surface deformation & MRI \\
{$[39]$} & GPU-K Dimensional tree algorithm, 3D edge registration & MRI \\
{$[41]$} & Random regression forest method & T1 weighted MRI \\
{$[42]$} & Curve fitting method & T1, T2 and PD Weighted MRI \\
\hline
\end{tabular}

\subsection{Other Methods and Approaches}

The morphological asymmetry is associated with functional variations in human brain populations, and some pathology is also strongly linked with abnormalities of brain symmetry/asymmetry [43]. In general, the human brain presents a high level of symmetry, but it is not perfectly symmetrical. Morphological and functional difference between the hemispheres makes the brain slightly asymmetrical. Different aspects of anatomical symmetry/asymmetry of the human brain were reported in a number of works. For example, Minoshima et al. [44] developed a bilateral reduction of metabolic activity in parietal, temporal and prefrontal regions for diagnosing Alzheimer's disease based on surface projection in PET brain images.

A method for analysis and visualization of cerebral brain asymmetry was reported by Marias et al. [45], who used a linear snake modal to extract fissure lines in each slice, and then fit a plane to these lines by orthogonal regression in MRI and CT brain images. The main advantage of these 
feature based methods is that they are robust to abnormalities and morphological inter-hemispheric differences because they do not assume symmetry. However, some of these existing approaches are sensitive to the outliers in the extracted features.

Blatter et al. [46] developed an intensity gradient based method to detect gross volumetric asymmetries in hemispheres in total, brain compartments and also different intracranial structures from the MRI brain images. Mangin et al. [47] applied the shape bottleneck algorithm and detected MSP to disconnect the left and right cerebral hemispheres (CH), cerebellum (CB), brainstem (BS) and various brain compartments in MR brain images. The hemispheric asymmetry in cerebral grey and white matter volumes from MR brain images was measured in the method reported in Maes et al. [48]. The grey and white matter segmentation was conducted through non-rigid registration with the labeled template image from the MR brain image, and the difference between grey matter volumes in left and right hemispheres was found. However, this automatic method is not applicable to neuroimages where a large lesion is present. Amunts et al. [49] found that an asymmetry in the depth of central sulcus has its relationship with handedness and gender in MR brain images. On other hand, there are several research groups who have also tried to quantitatively estimate brain asymmetries. Lee et al. [50] investigated hemispheric asymmetry and calculated the fractal dimension (FD) of the 3D skeletonized volume, which represented the cortical folding pattern using the measured volumes of gray matter and white matter and obtained the hemispheric asymmetries of each measurement from the MRI images.

A method proposed by Zhao et al. [18] is based on extended shape bottleneck algorithm and partial volume estimation. This method improved the accuracy of the brain hemispheres segmentation in 3D MRI brain volumes. Grigaitis and Meilunas [51] proposed a cellular neural networks method to analyze symmetry planes of brain images. This method detected the symmetrical plane by using the registration between hemispheres based on gray distribution from the binary image.

Zhao et al. [52] proposed an automatic novel method based on an Adaptive Disconnection method to segment the 3D MRI brain volume into the left and right cerebral hemispheres, the left and right cerebellum, and the brainstem by using the partial differential equations (PDE) and shape bottlenecks algorithm. This PDE algorithm detects and disconnects the shape bottlenecks between the wanted compartments without the aid of stereotaxic registration by using a partial volume estimation (PVE) algorithm.

A method proposed by Coupé et al. [53] is based on a nonlocal label fusion. The labels are obtained from multiple templates and are weighted according to the Euclidean distance between patch intensities. The brain anatomy segmentation accuracy was calculated using the patch size and number of training subjects. The result comparisons were carried out between the appearances based method and the template based method in MR brain images.

Romero et al. [54] presented an accurate and fast patch based multi template brain segmentation method, termed the NABS (Non-Local Automatic Brain Hemisphere Segmentation), for segmenting cerebral and cerebellar hemispheres and the brainstem from T1-weighted MR brain images. This NABS method was used to accurately delineate brain structures in healthy subjects across a wide range of ages. The main novelty of this new cost efficient segmentation method is the use of an optimized multi-label block-wise label fusion strategy that was designed especially to deal with the classification of main brain compartments, which significantly reduces the method complexity and is an extensive validation of this methodology.

The symmetry/asymmetry analysis using other methods and approaches along with the technique used and image modalities applied is summarized in Table 2. 
Table 2. Summary of the other methods and approaches for brain symmetric and asymmetric analysis.

\begin{tabular}{ccc}
\hline Methods & Techniques Used & Image Modality \\
\hline$[44]$ & Surface projection & PET \\
{$[45]$} & Linear Snake Modal, Orthogonal regression & MRI, CT \\
{$[46]$} & Intensity gradient based method & MRI \\
{$[47]$} & Shape bottleneck algorithm & MRI \\
{$[48]$} & Non rigid registration and template matching & MRI \\
{$[49]$} & Central sulcus measuring & 3D MRI \\
{$[50]$} & Fractal dimension & MRI \\
{$[18]$} & Extended shape bottleneck algorithm and partial volume estimate & 3D MRI \\
{$[51]$} & Content based cellular neural networks method and image registration & 3D, 2D MRI \\
{$[52]$} & Adaptive disconnection method & 3D MRI \\
{$[53]$} & Non localized label fusion and template & MRI \\
{$[54]$} & NABS method and patch based multi template segmentation & T1 Weighted MRI \\
\hline
\end{tabular}

Most of these existing methods are applicable to MRI of the brain and are also sensitive to image noises, imaging artifacts such as aliasing and orientation deviations [55]. Therefore, there is a greater need to develop an automatic, efficient and robust computational method to quantify the symmetry/asymmetry of human brain images from different imaging modalities for various biomedical and neuroscientific applications.

\section{Conclusions}

Brain symmetric/asymmetric analysis is an important tool and automatic methods for computer assisted diagnosis of many brain related disorders, such as schizophrenia, Alzheimer's disease, tumors, lesions, etc., require accurate inter-hemispheric segmentations. Moreover, to automate detection of the mid-sagittal plane (MSP) in various brain imagery, it is very useful to have a multitude of biomedical and neuroscientific applications. In this paper, we have analyzed and reviewed the existing computational methods developed and used for brain symmetric/asymmetric analysis with imagery obtained from different neuroimaging modalities. All of these existing methods are still in their infancy, and more efficient and robust methods need to be devised to work with the images of different imaging modalities. Moreover, due to limitations in the imaging mechanisms of the human brain, these methods need to be robust to noise and other imaging artifacts. The methods reviewed in this work utilize custom made datasets either produced in-house or adapted from other available neuroimaging datasets. Unfortunately, to the best of our knowledge, there are no dedicated datasets available for performing and benchmarking symmetric/asymmetric analysis. This requires dedicated manual drawing by multiple neuroscientists/clinicians. Generating standardized ground truth as well as making the automatic MSP methods as open source tools and utilizing fast graphical processing units (GPUs) and distributed computing acceleration can facilitate practical usage in the neuroimaging community.

Author Contributions: M. Senthamilselvi performed the initial literature survey under the supervision of P. Kalavathi. P. Kalavathi, Surya Prasath, and M. Senthamilselvi discussed and wrote the article. All authors revised the manuscript. All authors have read and approved the final manuscript.

Conflicts of Interest: The authors declare no conflict of interest.

\section{References}

1. Sonka, M.; Tadikonda, S.K.; Collins, S.M. Knowledge-based interpretation of MR brain images. IEEE Trans. Med. Imaging 1996, 15, 443-452.

2. Damasio, H. Human Brain Anatomy in Computerized Images, 2nd ed.; Oxford University Press: New York, NY, USA, 2005.

3. Wang, S.; Zhang, Y.; Yang, X.; Sun, P.; Dong, Z.; Liu, A.; Yuan, T.F. Pathological brain detection by a novel image feature-fractional Fourier entropy. Entropy 2015, 17, 8278-8296. 
4. Wang, S.; Lu, S.; Dong, Z.; Yang, J.; Yang, M.; Zhang, Y. Dual-tree complex wavelet transform and twin support vector machine for pathological brain detection. Appl. Sci. 2016, 6, 169, doi:10.3390/app6060169.

5. MRIMASTER.COM. Available online: https://mrimaster.com/anatomybrainsagittal.html (accessed on 18 April 2017).

6. Kalavathi, P.; Prasath, V.B.S. Methods on skull stripping of MRI head scan images-A review. J. Digit. Imaging 2016, 29, 365-379.

7. Toga, A.W.; Thompson, P.M. Mapping brain asymmetry. Nat. Rev. Neurosci. 2003, 4, 37-48.

8. Nielsen, J.A.; Zielinski, B.A.; Ferguson, M.A.; Lainhart, J.E.; Anderson, J.S. An evaluation of the left-brain vs. right-brain hypothesis with resting state functional connectivity magnetic resonance imaging. PLoS ONE 2013, 8, e71275.

9. Harisson, P. The neuropathology of schizophrenia: A critical review of the data and their interpretation. Brain 1999, 122, 593-624.

10. Thompson, P.; Moussai, J.; Zohoori, S.; Goldkorn, A.; Khan, A.; Mega, M.; Small, G.; Cummings, J.; Toga, A. Cortical variability and asymmetry in normal aging and Alzheimer's disease. Cereb. Cortex 1998, 8, 492-509.

11. Herbert, M.R.; Ziegler, D.; Deutsch, C.; O’Brien, L.; Kennedy, D.; Filipek, P.; Bakardjiev, A.; Hodgson, J.; Takeoka, M.; Makris, N.; et al. Brain asymmetries in autism and developmental language disorder: A nested whole-brain analysis. Brain 2005, 128, 213-226.

12. Bilder, R.M.; Wu, H.; Bogerts, B.; Degreef, G.; Ashtari, M.; Alvir, J.M.; Snyder, P.J.; Lieberman, J.A. Absence of regional hemispheric volume asymmetries in first-episode schizophrenia. Am. J. Psychiatry 1994, 151, 1437-1447.

13. Pepe, A.; Zhao, L.; Koikkalainen, J.; Hietala, J.; Ruotsalainen, U.; Tohka, J. Automatic statistical shape analysis of cerebral asymmetry in 3D T1-weighted magnetic resonance images at vertex-level: Application to neuroleptic-naïve schizophrenia. Magn. Reson. Imaging 2013, 31, 676-687.

14. Sarmiento, C.I.; Prasath, V.B.S. Design of a Novel Servo-motorized Laser Device for Visual Pathways Diseases Therapy. BRAIN. Broad Res. Artif. Intell. Neurosci. 2015, 6, 84-90.

15. Wang, S.; Yang, M.; Zhang, Y.; Li, J.; Zou, L.; Lu, S.; Liu, B.; Yang, J.; Zhang, Y. Detection of left-sided and right-sided hearing loss via fractional Fourier transform. Entropy 2016, 18, 194.

16. Jayasuriya, S.A.; Liew, A.W.C. Fractal dimension as a symmetry measure in 3D brain MRI analysis. In Proceedings of the IEEE International Conference on Machine Learning and Cybernetics, Xi'an, China, 15-17 July 2012; pp. 1118-1123.

17. Boykov, Y.; Veksler, O.; Zabih, R. Fast approximate energy minimization via graph cuts. IEEE Trans. Pattern Anal. Mach. Intell. 2001, 23, 1222-1239.

18. Zhao, L.; Tohka, J.; Ruotsalainen, U. Accurate 3D left-right brain hemisphere segmentation in MR images based on shape bottlenecks and partial volume estimation. In Proceedings of the 15th Scandinavian Conference on Image Analysis, SCIA'07, Aalborg, Denmark, 10-14 June 2007; pp. 581-590.

19. Brummer, M.E. Hough transform detection of the longitudinal fissure in tomographic head images. IEEE Trans. Med. Imaging 1991, 10, 74-81.

20. Ruppert, G.; Teverovskiy, L.; Yu, C.P.; Falcao, A.X.; Liu, Y. A new symmetry-based method for mid-sagittal plane extraction in neuroimages. In Proceedings of the IEEE International Symposium on Biomedical Imaging, Chicago, IL, USA, 30 March-2 April 2011; pp. 285-288.

21. Ardekani, B.A.; Kershaw, J.; Braun, M.; Kanuo, I. Automatic detection of the mid-sagittal plane in 3-D brain images. IEEE Trans. Med. Imaging 1997, 16, 947-952.

22. Liu, Y.; Collins, R.T.; Rothfus, W.E. Robust midsagittal plane extraction from normal and pathological 3-D neuroradiology images. IEEE Trans. Med. Imaging 2001, 20, 175-192.

23. Prima, S.; Ourselin, S.; Ayache, N. Computation of the mid-sagittal plane in 3-D brain images. IEEE Trans. Med. Imaging 2002, 21, 122-138.

24. Brett, M.; Johnsrude, I.S.; Owen, A.M. The problem of functional localization in the human brain. Nat. Rev. Neurosci. 2002, 3, 243-249.

25. Tuzikov, A.V.; Colliot, O.; Bloch, I. Brain symmetry plane computation in MR images using inertia axes and optimization. In Proceedings of the International Conference on Pattern Recognition, Québec City, QC, Canada, 11-15 August 2002; Volume 1, pp. 516-519.

26. Tuzikov, A.V.; Colliot, O.; Bloch, I. Evaluation of the symmetry plane in 3D MR brain images. Pattern Recognit. Lett. 2003, 24, 2219-2233. 
27. Hu, Q.; Nowinski, W.L. A rapid algorithm for robust and automatic extraction of the midsagittal plane of the human cerebrum from neuroimages based on local symmetry and outlier removal. NeuroImage 2003, 20, 2153-2165.

28. Ekin, A. Feature-based brain mid-sagittal plane detection by RANSAC. In Proceedings of the European Signal Processing Conference, Florence, Italy, 4-8 September 2006; pp. 1-4.

29. Volkau, I.; Prakash, B.; Ananthasubramaniam, A.; Gupta, V.; Aziz, A.; Nowinski, W.L. Quantitative analysis of brain asymmetry by using the divergence measure: normal-pathological brain discrimination. Acad. Radiol. 2006, 13, 752-758.

30. Liang, L.; Rehm, K.; Woods, R.P.; Rottenberg, D.A. Automatic segmentation of left and right cerebral hemispheres from MRI brain volumes using the graph cuts algorithm. NeuroImage 2007, 34, 1160-1170.

31. Song, E.; Wang, Q.; Ma, G.; Liu, H. Symmetry analysis to detect pathological brain in MRI. In Proceedings of the International Symposium on Multispectral Image Processing and Pattern Recognition, Wuhan, China, 15-17 November 2007; p. 67891F.

32. Liu, S.X.; Imielinska, C.; Laine, A.; Millar, W.S.; Connolly, E.S.; D'Ambrosio, A.L. Asymmetry analysis in rodent cerebral ischemia models. Acad. Radiol. 2008, 15, 1181-1197.

33. Bergo, F.P.; Falcão, A.X.; Yasuda, C.L.; Ruppert, G.C. Fast, accurate and precise mid-sagittal plane location in 3D MR images of the brain. In Proceedings of the Biomedical Engineering Systems and Technologies, Funchal, Madeira, Portugal, 28-31 January 2008; pp. 278-290.

34. Zhou, L.; Dupont, P.; Baete, K.; Van Paesschen, W.; Van Laere, K.; Nuyts, J. Detection of inter-hemispheric metabolic asymmetries in FDG-PET images using prior anatomical information. NeuroImage 2009, 44, 35-42.

35. Teverovskiy, L.; Li, Y. Truly 3D midsagittal plane extraction for robust neuroimage registration. In Proceedings of the IEEE International Symposium on Biomedical Imaging, Arlington, VA, USA, 6-9 April 2006; pp. 860-863.

36. Qi, X.; Belle, A.; Shandilya, S.; Chen, W.; Cockrell, C.; Tang, Y.; Ward, K.R.; Hargraves, R.H.; Najarian, K. Ideal midline detection using automated processing of brain CT image. Open J. Med. Imaging 2013, 3, 51-53.

37. Favretto, F.O.; Bergo, F.P.; Falcao, A.X. A fast and automatic method for 3D rigid registration of MR images of the human brain. In Proceedings of the Brazilian Symposium on Computer Graphics and Image Processing (SIBGRAPI), Campo Grande, Brazil, 12-15 October 2008; pp. 121-128.

38. Kuijf, H.J.; van Veluw, S.J.; Geerlings, M.I.; Viergever, M.A.; Biessels, G.J.; Vincken, K.L. Automatic Extraction of the Midsagittal Surface from Brain MR Images using the Kullback-Leibler Measure. Neuroinformatics 2014, 12, 395-403.

39. Wu, H.; Wang, D.; Shi, L.; Wen, Z.; Ming, Z. Midsagittal plane extraction from brain images based on 3D SIFT. Phys. Med. Biol. 2014, 59, 1367.

40. Wu, H.; Wang, D.; Shi, L.; Wen, Z.; Ming, Z. Fast and robust symmetry detection for brain images based on parallel scale-invariant feature transform matching and voting. Int. J. Imaging Syst. Technol. 2013, 23, 314-326.

41. Liu, Y.; Dawant, B.M. Automatic Localization of the Anterior Commissure, Posterior Commissure, and Midsagittal Plane in MRI Scans using Regression Forests. IEEE J. Biomed. Health Inform. 2015, 19, 1362-1374.

42. Kalavathi, P.; Prasath, V.B.S. Automatic segmentation of cerebral hemispheres in MR human head scans. Int. J. Imaging Syst. Technol. 2016, 26, 15-23.

43. Zhang, Y.; Dong, Z.; Wang, S.; Ji, G.; Yang, J. Preclinical diagnosis of magnetic resonance (MR) brain images via discrete wavelet packet transform with Tsallis entropy and generalized eigenvalue proximal support vector machine (GEPSVM). Entropy 2015, 17, 1795-1813.

44. Minoshima, S.; Frey, K.A.; Koeppe, R.A.; Foster, N.L.; Kuhl, D.E. A diagnostic approach in Alzheimer's disease using three-dimensional stereotactic surface projections of fluorine-18-FDG PET. J. Nuclear Med. 1995, 36, 1238-1248.

45. Marias, P.; Guillernaud, R.; Sakuma, M.; Zisserman, A.; Brady, M. Visualising cerebral asymmetry. In Visualization in Biomedical Computing; Springer: Berlin/Heidelberg, Germany, 1996; pp. 411-416.

46. Blatter, D.D.; Bigler, E.D.; Johnson, C.S.; Anderson, C.; Gale, S.D. A normative database from magnetic resonance imaging. In Neuroimaging I; Springer: Berlin/Heidelberg, Germany, 1996; pp. 79-95.

47. Mangin, J.F.; Régis, J.; Frouin, V. Shape bottlenecks and conservative flow systems. In Proceedings of the Workshop on Mathematical Methods in Biomedical Image Analysis; San Francisco, CA, USA, 21-22 June 1996; pp. 319-328. 
48. Maes, F.; Van Leemput, K.; DeLisi, L.E.; Vandermeulen, D.; Suetens, P. Quantification of cerebral grey and white matter asymmetry from MRI. In Proceedings of the Medical Image Computing and Computer-Assisted Intervention (MICCAI), Cambridge, UK, 19-22 September 1999; pp. 348-357.

49. Amunts, K.; Jäncke, L.; Mohlberg, H.; Steinmetz, H.; Zilles, K. Interhemispheric asymmetry of the human motor cortex related to handedness and gender. Neuropsychologia 2000, 38, 304-312.

50. Lee, J.M.; Yoon, U.; Kim, J.J.; Kim, I.Y.; Lee, D.S.; Kwon, J.S.; Kim, I.Y. Analysis of the hemispheric asymmetry using fractal dimension of a skeletonized cerebral surface. IEEE Trans. Biomed. Eng. 2004, 51, 1494-1498.

51. Grigaitis, D.; Meilunas, M. Automatic extraction of symmetry plane from falx cerebri areas in CT slices. In Bildverarbeitung für die Medizin; Springer: Berlin/Heidelberg, Germany, 2007; pp. 267-271.

52. Zhao, L.; Ruotsalainen, U.; Hirvonen, J.; Hietala, J.; Tohka, J. Automatic cerebral and cerebellar hemisphere segmentation in 3D MRI: adaptive disconnection algorithm. Med. Image Anal. 2010, 14, 360-372.

53. Coupé, P.; Manjón, J.V.; Fonov, V.; Pruessner, J.; Robles, M.; Collins, D.L. Patch-based segmentation using expert priors: Application to hippocampus and ventricle segmentation. NeuroImage 2011, 54, 940-954.

54. Romero, J.E.; Manjón, J.V.; Tohka, J.; Coupé, P.; Robles, M. NABS: non-local automatic brain hemisphere segmentation. Magn. Reson. Imaging 2015, 33, 474-484.

55. Prasath, V.B.S.; Kalavathi, P. Adaptive nonlocal filtering for brain MRI restoration. In Proceedings of the Second International Symposium on Signal Processing and Intelligent Recognition Systems (SIRS), Trivandrum, Kerala, India, 16-19 December 2015; Thampi, S.M., Bandyopadhyay, S., Krishnan, S., Li, K.-C., Mosin, S., Ma, M., Eds.; Springer AISC: Chennai, India; pp. 571-580.

(C) 2017 by the authors. Licensee MDPI, Basel, Switzerland. This article is an open access article distributed under the terms and conditions of the Creative Commons Attribution (CC BY) license (http:/ / creativecommons.org/licenses/by/4.0/). 